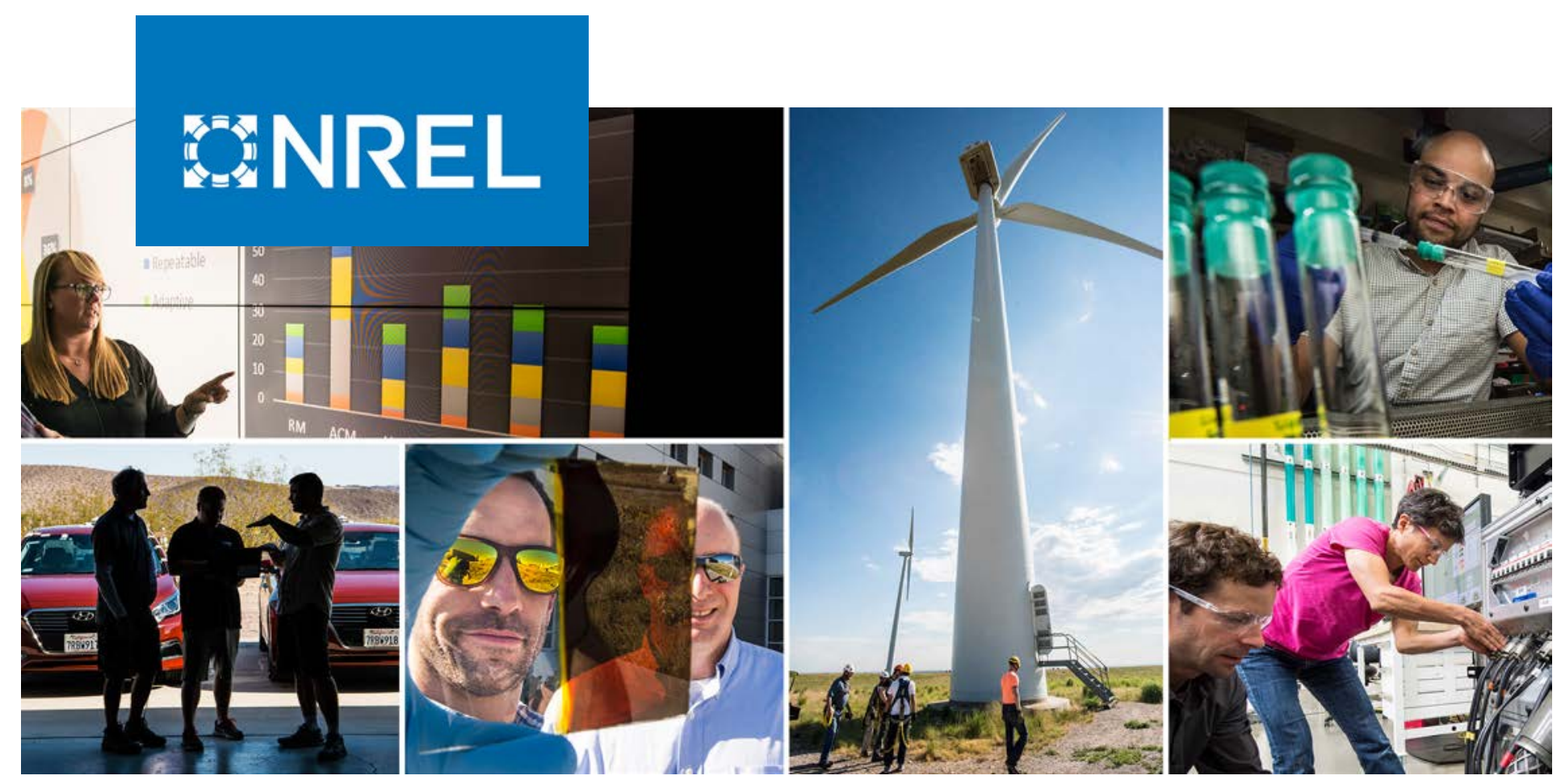

\title{
Hardware-in-the-Loop Test Bed and Test Methodology for Microgrid Controller Evaluation
}

\section{Preprint}

\author{
Kumaraguru Prabakar, ${ }^{1}$ Annabelle Pratt, ${ }^{1}$ \\ Dheepak Krishnamurthy, ${ }^{1}$ and Arindam Maitra ${ }^{2}$
}

\section{National Renewable Energy Laboratory \\ 2 Electric Power Research Institute}

\author{
Presented at the 2018 IEEE/PES Transmission and Distribution \\ Conference and Exposition (T\&D) \\ Denver, Colorado \\ April 16-19, 2018
}

\begin{abstract}
(C) 2018 IEEE. Personal use of this material is permitted. Permission from IEEE must be obtained for all other uses, in any current or future media, including reprinting/republishing this material for advertising or promotional purposes, creating new collective works, for resale or redistribution to servers or lists, or reuse of any copyrighted component of this work in other works. See K. Prabakar, A. Pratt, D. Krishnamurthy and A. Maitra, "Hardware-in-the-Loop Test Bed and Test Methodology for Microgrid Controller Evaluation," 2018 IEEE/PES Transmission and Distribution Conference and Exposition (T\&D), Denver, CO, 2018, pp. 1-9. doi: 10.1109/TDC.2018.8440246
\end{abstract}

NREL is a national laboratory of the U.S. Department of Energy

Office of Energy Efficiency \& Renewable Energy

Operated by the Alliance for Sustainable Energy, LLC

This report is available at no cost from the National Renewable Energy Laboratory (NREL) at www.nrel.gov/publications.

\section{Conference Paper}

NREL/CP-5D00-73627

May 2019 


\title{
GNREL
}

\section{Hardware-in-the-Loop Test Bed and Test Methodology for Microgrid Controller Evaluation}

\section{Preprint}

\author{
Kumaraguru Prabakar, ${ }^{1}$ Annabelle Pratt, ${ }^{1}$ \\ Dheepak Krishnamurthy, ${ }^{1}$ and Arindam Maitra ${ }^{2}$ \\ 1 National Renewable Energy Laboratory \\ 2 Electric Power Research Institute
}

\section{Suggested Citation}

Prabakar, Kumaraguru, Annabelle Pratt, Dheepak Krishnamurthy, and Arindam Maitra. 2019. Hardware-in-the-Loop Test Bed and Test Methodology for Microgrid Controller Evaluation: Preprint. Golden, CO: National Renewable Energy Laboratory. NREL/CP-5D00-73627. https://www.nrel.gov/docs/fy19osti/73627.pdf.

NREL is a national laboratory of the U.S. Department of Energy Office of Energy Efficiency \& Renewable Energy Operated by the Alliance for Sustainable Energy, LLC

This report is available at no cost from the National Renewable Energy Laboratory (NREL) at www.nrel.gov/publications.

Contract No. DE-AC36-08GO28308
Conference Paper NREL/CP-5D00-73627 May 2019

National Renewable Energy Laboratory 15013 Denver West Parkway Golden, CO 80401 303-275-3000 • www.nrel.gov 


\section{NOTICE}

This work was authored [in part] by the National Renewable Energy Laboratory, operated by Alliance for Sustainable Energy, LLC, for the U.S. Department of Energy (DOE) under Contract No. DE-AC36-08GO28308. Funding provided by the U.S. Department of Energy Office of Energy Efficiency and Renewable Energy. The views expressed herein do not necessarily represent the views of the DOE or the U.S. Government. The U.S. Government retains and the publisher, by accepting the article for publication, acknowledges that the U.S. Government retains a nonexclusive, paid-up, irrevocable, worldwide license to publish or reproduce the published form of this work, or allow others to do so, for U.S. Government purposes.

This report is available at no cost from the National Renewable Energy Laboratory (NREL) at www.nrel.gov/publications.

U.S. Department of Energy (DOE) reports produced after 1991 and a growing number of pre-1991 documents are available free via www.OSTI.gov.

Cover Photos by Dennis Schroeder: (clockwise, left to right) NREL 51934, NREL 45897, NREL 42160, NREL 45891, NREL 48097, NREL 46526.

NREL prints on paper that contains recycled content. 


\section{Hardware-in-the-Loop Test Bed and Test Methodology for Microgrid Controller Evaluation}

\author{
Kumaraguru Prabakar, Annabelle Pratt, Dheepak \\ Krishnamurthy \\ National Renewable Energy Laboratory \\ kumaraguru.prabakar@nrel.gov, annabelle.pratt@nrel.gov, \\ dheepak.krishnamurthy@nrel.gov
}

\author{
Arindam Maitra \\ Electric Power Research Institute \\ amaitra@epri.com
}

\begin{abstract}
Increasing penetration levels of distributed energy resources are making the deployment of microgrids more feasible. Controllers that operate such microgrids are key to realizing the objectives of the microgrid owner or operator and there is a need to evaluate microgrid controller performance prior to field deployment. This paper describes a controller hardware-in-the-loop and power hardware-in-the-loop microgrid controller test bed that was designed and constructed to evaluate the capabilities of a microgrid controller for a proposed campus microgrid. This paper also presents a test methodology to evaluate microgrid controller functionality, and it describes how the controller was assessed through the application of different test scenarios. Results from the testing are presented to provide insight into the capabilities of the test bed.
\end{abstract}

Keywords-Controller hardware-in-the-loop, controller test bed, hardware-in-the-loop, microgrid, power hardware-in-the-loop.

\section{INTRODUCTION}

The pace of integrating photovoltaic (PV) systems and battery energy storage systems (BESS) continues to accelerate as costs drop and more cities and states mandate higher percentages of power from renewable sources. The proliferation of these distributed energy resources (DERs) is making microgrids more feasible. Microgrids are capable of islanding facilities, campuses, communities, or even entire distribution feeders from the utility grid while sustaining loads. This can improve reliability and relieve stress on the utility grid during abnormal conditions [1] [2].

In 2014, the U.S. Department of Energy, Office of Electricity Delivery and Energy Reliability, undertook several projects to promote the integration of microgrids [3]. Some were initiated through funding opportunity announcement (FOA) DE-FOA-0000997 (FOA 997), which supports the development and testing of advanced, commercial-grade microgrid controllers that can manage aggregated generation capacity from 1-10 MW. As a key component of a microgrid system, the controller manages assets to meet the microgrid's objectives [4]. Ensuring the supply of power to loads during islanded operation is the primary objective. Other objectives might include reducing energy costs, maximizing the use of renewable DERs, and limiting greenhouse gas emissions.

This work was supported by the U.S. Department of Energy under Contract No. DE-AC36-08GO28308 with Alliance for Sustainable Energy, LLC, the Manager and Operator of the National Renewable Energy Laboratory.
Microgrid controllers might also be required to interface with distribution management systems or other higher-level entities - e.g., DER aggregators - to provide grid services.

The test bed described in this paper was designed to support an FOA 997-funded project led by the Electric Power Research Institute (EPRI) that focused on a proposed microgrid for the Buffalo Niagara Medical Campus (BNMC) in Buffalo, New York. It can be used to test the capabilities of a microgrid controller before it is installed in the field. A microgrid controller test bed can be set up with software simulation capability only, controller hardware-in-the-loop (CHIL) only, controller and power hardware-in-the-loop (CHIL/PHIL), or hardware only [4]. A hardware-in-the-loop (HIL) test bed can be used to evaluate a controller for a specific microgrid site or a generic microgrid system, an approach often used to compare general capabilities of different controllers. We used a sitespecific CHIL/PHIL test bed with the actual microgrid controller hardware for CHIL and battery inverter hardware for PHIL. This allowed us to reduce modeling inaccuracies, especially because proprietary controls embedded within the hardware cannot always be accurately modeled. This paper also proposes a test method and describes test cases that can be used to evaluate the performance of a microgrid controller. The evaluation of microgrid controllers prior to field deployment benefits microgrid controller providers, developers, and utilities.

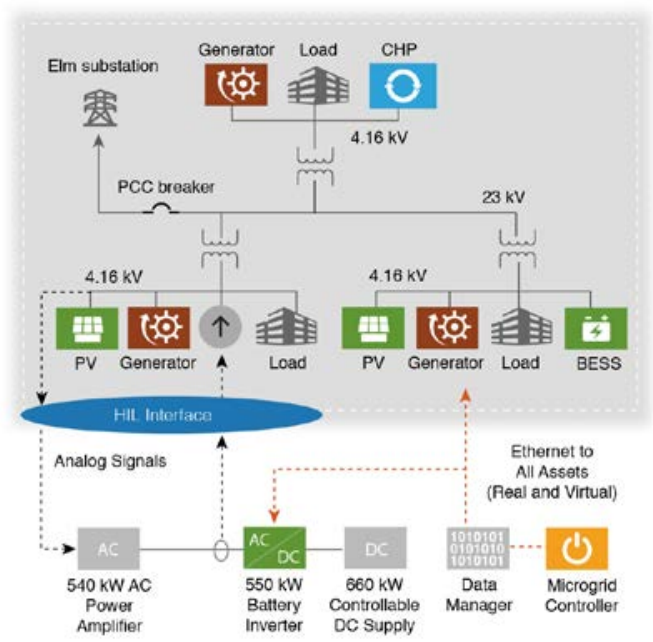

Fig. 1. Proposed microgrid system model for BNMC 


\section{TEST BED SETUP}

\section{A. Real-Time Power System Model}

The power system model of the proposed BNMC microgrid was simulated using an RTDS digital real-time simulator with a time step of 190 usec. Fig. 1 is a simplified diagram of the model. The proposed microgrid will include three medical campuses with their own backup generation. The coincident peak load for the microgrid is $24 \mathrm{MW}$. Table I lists the existing and proposed microgrid assets used in the simulation. The microgrid controller controls the real and reactive power flow across the point of common coupling (PCC) breaker as well as the operating modes of all assets based on whether the microgrid is grid-connected, islanded, or transitioning between these two states.

TABLE I. MiCROGRID ASSETS

\begin{tabular}{|l|c|l|}
\hline \multicolumn{1}{|c|}{ Asset Type } & $\begin{array}{c}\text { Number of } \\
\text { assets }\end{array}$ & \multicolumn{1}{|c|}{ Rating } \\
\hline Diesel generator & 2 & $8 \mathrm{MW}$ and 7 MW \\
\hline Natural gas generator & 2 & $5 \mathrm{MW}$ each \\
\hline Combined heat and power (CHP) & 1 & $7.7 \mathrm{MW}$ \\
\hline PV (non-dispatchable) & 2 & $0.36 \mathrm{MW}$ and $0.26 \mathrm{MW}$ \\
\hline BESS & 1 & $50 \mathrm{~kW}$ \\
\hline
\end{tabular}

\section{B. Controller Hardware-in-the-Loop Setup}

The test bed can use a variety of standard industry communication protocols supported by microgrid controllers and DER assets. The microgrid controller under test, Spirae's Wave $^{\circledR}$ software control platform, uses Modbus and DNP3. It was set up to send most of the signals using Modbus, and others using DNP3. In the field, a data manager-which can also serve as a protocol translator when required - is used to acquire signals from the controller and send them to individual assets and vice versa. We included a data manager in our laboratory setup to mimic the field setup, as shown in Fig. 2.

At the time of testing, it was faster and computationally more efficient to use DNP3 in the RDTS than to use Modbus to send and receive information. Thus, RTDS sends information using DNP3, and we used the data manager to translate the signals between the RTDS and the controller. The data exchanged includes information such as root-mean-square voltage, frequency, circuit breaker status/control, and generator mode of operation and status/control. The microgrid controller required more than 6,500 data points to be sent/received using DNP3/Modbus protocols.

\section{Power Hardware-in-the-Loop Setup}

One BESS asset was included in the microgrid test bed through a PHIL setup. The BESS consists of a $660-\mathrm{kW}$

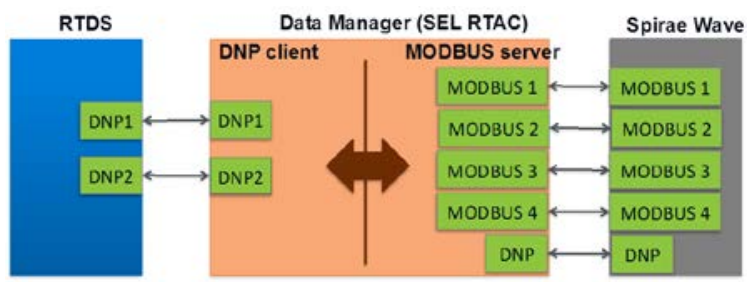

Fig. 2. Ethernet-based data communication between the RTDS and microgrid controller through the data manager.

bidirectional, controllable DC source that emulates the battery; a $540-\mathrm{kW}$ bidirectional, controllable AC source; and a 550-kW Schneider battery inverter as shown in Fig. 1. More information regarding the PHIL experimental setup can be found in [5]. The simulated three-phase voltage waveform from the bus connected to the BESS asset was used to control the AC source through analog outputs from the RTDS. The analog signals were amplified by the AC source to power the battery inverter. The real and reactive power set points for charging and discharging the battery were determined by the microgrid controller. The PHIL interface was designed based on our prior work described in [6] to ensure stability and accuracy.

\section{EVALUATION APPROACH}

Currently, no standard exists for evaluating microgrid controllers, although a draft version of the IEEE P2030.8, standard for the testing of microgrid controllers has been developed [6]. Thus, this paper proposes an approach for evaluating the functional performance of a microgrid controller. Similarly, no standard exists that defines the required functions of microgrid controllers, although a draft version of the IEEE P2030.7 standard for the specification of microgrid controllers has been developed. Thus, we used the following functional requirements outlined in FOA 997: C1 (Disconnection), C2 (Resynchronization and Reconnection), C3 (Steady-State Frequency Range, Voltage Range, and Power Quality), C4 (Protection), and C5 (Dispatch). Four HIL test scenarios were developed, and the results were compared against the requirements defined in FOA 997, as shown in Table II. For each scenario, test cases were simulated, and some of the results are presented in the next section.

\section{TEST CASE DESCRIPTIONS AND RESUlts}

Two test cases were simulated for Scenario A-normal grid-connected operation with and without power dispatch-to validate that the microgrid controller can successfully dispatch microgrid assets while grid-connected. For both test cases, the PCC circuit breaker was closed and the microgrid was connected to the utility grid, which effectively controls the microgrid's voltage and frequency.

TABLE II. MAPPING OF SCENARIOS To FunCtional REQUiREMENTS

\begin{tabular}{lcrrrr}
\hline \multicolumn{1}{c}{ Scenario Description } & C1 & C2 & C3 & C4 & C5 \\
\hline & Disconnection & Resynchronization and Reconnection & Steady-State & Protection & Dispatch \\
\hline $\begin{array}{l}\text { A. Operating While Connected to the Utility } \\
\text { B. Separating from the Utility }\end{array}$ & $\mathrm{X}$ & & $\mathrm{X}$ \\
$\begin{array}{l}\text { C. Operating While Separated from the Utility } \\
\text { D. Connecting to the Utility }\end{array}$ & & $\mathrm{X}$ & $\mathrm{X}$ & $\mathrm{X}$ \\
\hline
\end{tabular}




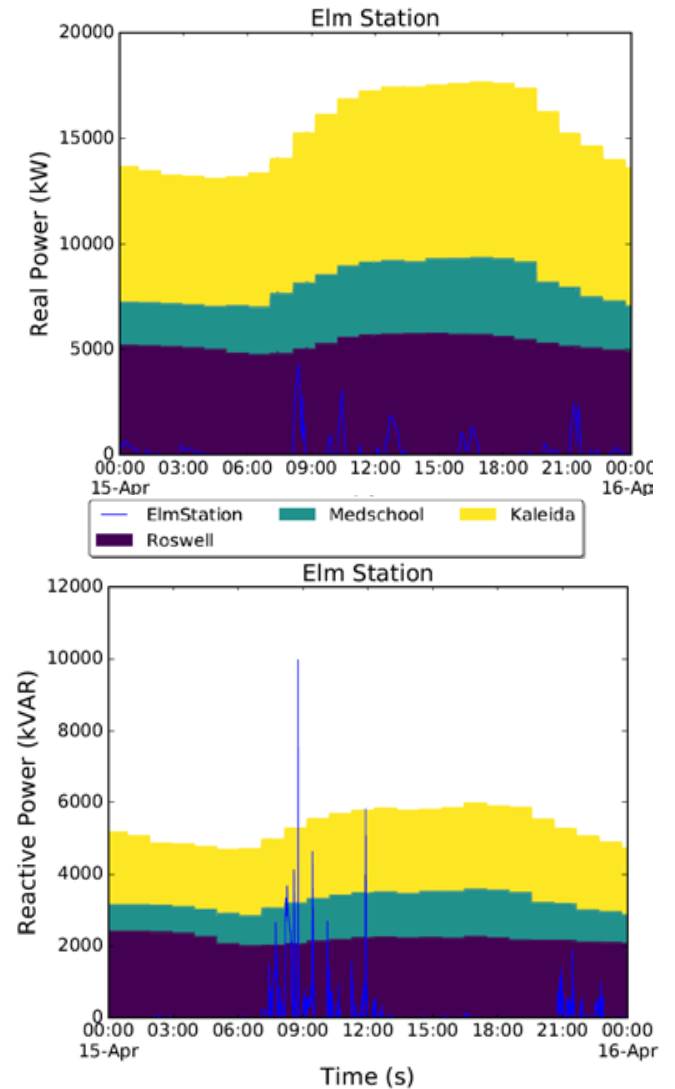

Fig. 3. Scenario A loads and test case A2 PCC power flow

Load profiles were applied based on historical data for Tuesday, April 15, 2014. This represents a weekday with a significant load of approximately $18 \mathrm{MW}$. The nondispatchable generation, which is all PV for this site, was determined by simulating the PV panels in the RSCAD software with insolation data available at the National Renewable Energy Laboratory. Accelerated (faster than real-time) simulations were performed whereby 2 minutes of simulation time equaled 60 minutes of real time. We selected 2 minutes to allow transients to settle after a load step.

\section{A. Normal Grid-Connected Operation with No Dispatch (Test Case A1)}

In this case, the Wave microgrid controller's dispatch function was disabled, and all dispatchable generation was turned off. The same load profiles were used for test cases A1 and A2, and the load profiles of all three campuses can be seen in Fig. 3 as stacked plots. For test case A1, the PCC power is slightly greater than the sum of the load powers because of distribution losses within the BNMC.

\section{B. Normal Grid-Connected Operation with Dispatch (Test Case A2)}

This case was set up similarly to A1, except that the microgrid controller was set to dispatch based on cost parameters (startup, hourly, operating, stopping, etc.) to achieve the active and reactive set points for PCC power flow. As an example, the PCC power flow set points were set to zero for both active and reactive power. The load of all the campuses within the BNMC and the power flows through the PCC circuit breaker are shown as stacked plots in Fig. 3. The
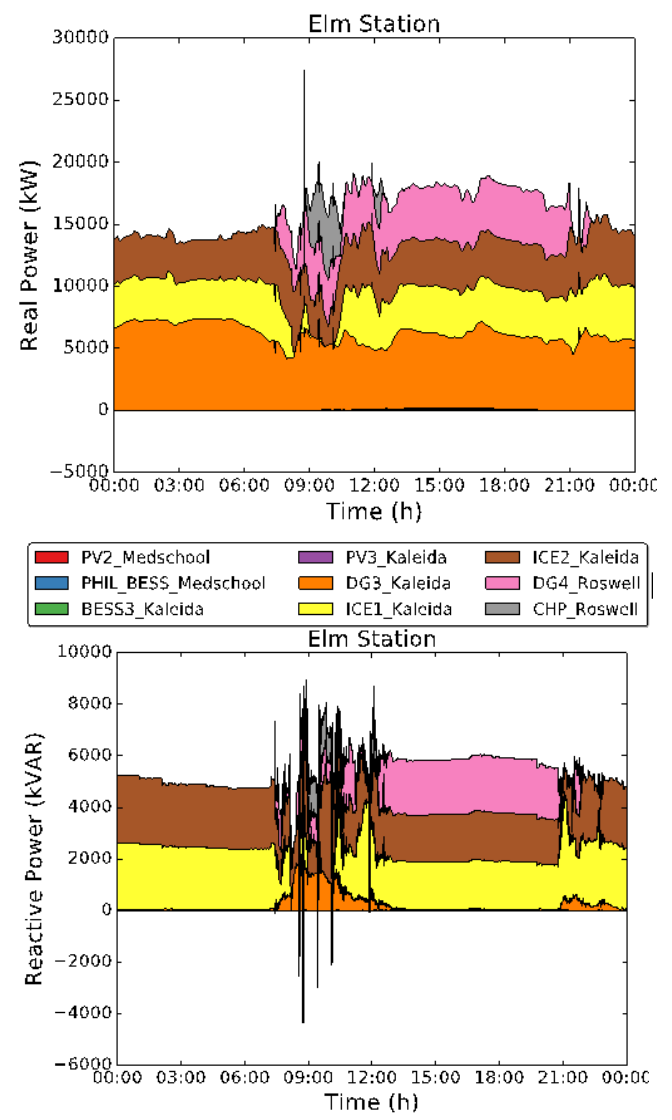

Fig. 4. Test case A2 generation

power flow through the PCC circuit breaker is nonzero for short periods after a load step until the microgrid controller is able to regulate it to zero. The generation dispatched by the microgrid controller to regulate the PCC power flow to zero is shown as stacked plots in Fig. 4. The microgrid controller dispatched internal combustion engines ICE1 and ICE2 and diesel generator DG3 for the baseload, and during peak demand it dispatched diesel generator DG4 and the CHP unit. Once the demand dropped in the evening, DG4 was turned off for cost benefits. The oscillations in real and reactive power during peak load were mainly caused by startup and shut down transients of assets.

The performance of the microgrid controller was evaluated according to the following criteria set forth in FOA 997: (1) survivability, (2) economic operation, and (3) environmental performance. Fig. 4 shows that survivability was achieved because all loads were served. Fig. 5 shows the cost comparison between A1 and A2. The cumulative cost shows that during grid-connected operation, the cost was lower for A2 than for A1. The results were similar for environmental performance, with lower emissions for A2 than for A1. The Wave microgrid controller can be configured with different optimizers, including lowest cost or emissions. In these tests, a reliability heuristic was used, so the optimization results were determined based on the ratios of utility to diesel and natural gas prices and emissions.

Three test cases were simulated for scenario B to validate the ability of the Wave microgrid controller to successfully island the microgrid under planned and unplanned conditions. 


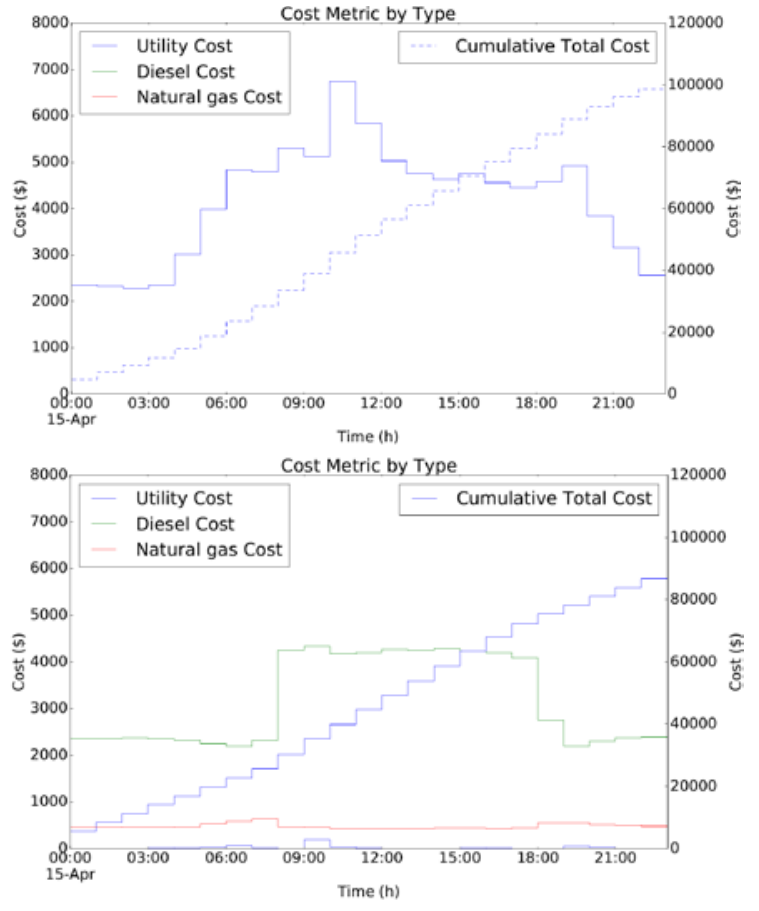

Fig. 5. Cost comparison of $\mathrm{A} 1$ and $\mathrm{A} 2$

\section{Planned Separation (Test Case B1)}

The utility grid remained within normal operating conditions, and the microgrid separation was part of a planned operation. We verified that the Wave microgrid controller set DG3 as the voltage/frequency master and that the microgrid operated for several minutes after islanding to confirm stable operation. Simulation results for a planned islanding event are shown in [4] that confirm that a smooth transition occurred.

\section{Unplanned Separation Due to an External Fault (Test}

Case B2) and Unplanned Separation Due to Loss of Utility (Test Case B3)

Functional requirement $\mathrm{C} 4$ states that the microgrid controller should separate from the utility in response to an external fault or loss of utility power. In B2, an external singlephase-to-ground fault was applied on Phase A, and in B3 the grid voltage source was set to zero to simulate a grid outage. The Wave microgrid controller did not control any protective devices in either case, which is typical for microgrid controllers; rather, it relied on other assets within the microgrid to provide protection functions.

We implemented controls in the simulated PCC circuit breaker that islanded the microgrid from the main grid in response to the fault and grid outage. Once the microgrid was islanded, we verified that the microgrid controller set DG3 to be the voltage/frequency master and that the microgrid operated in islanded mode for several minutes.

Two test cases were simulated for scenario $\mathrm{C}$ to validate the ability of the Wave microgrid controller to dispatch assets to achieve successful islanded operation. Only the first test case, for normal islanded operation, is presented here. The second, $\mathrm{C} 2$, simulated an internal short during islanded operation, and results are not presented because the microgrid controller did not provide protective functions.

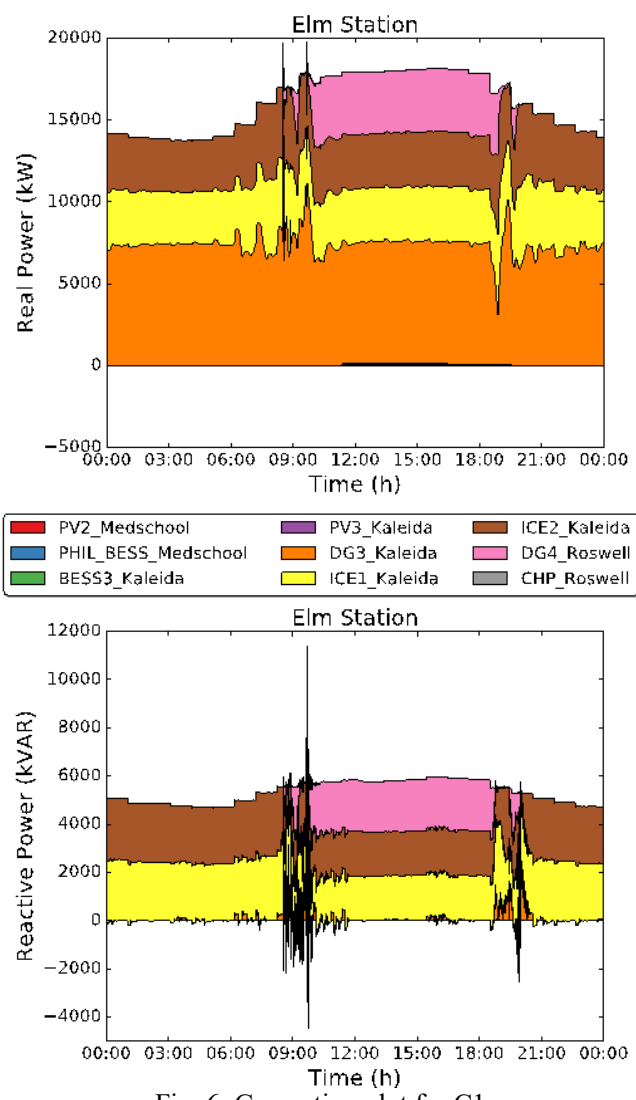

Fig. 6. Generation plot for $\mathrm{C} 1$

\section{E. Normal Islanded Operation (Test Case C1)}

The microgrid was set up to operate in islanded mode through a planned islanding event as described in B1. Once islanded, the same load and solar insolation profiles used for A2 were programmed. The generation profiles of all generation - dispatchable and nondispatchable - are shown as a stacked plot in Fig. 6. The generation dispatch is similar to that for A2, except that the microgrid controller selected not to dispatch the CHP unit. The microgrid controller successfully operated the microgrid in islanded mode under normal conditions by dispatching generation to continuously serve all loads within the microgrid. The oscillations that occur during peak hours are due to the transient behavior of the assets at startup and shut down.

One test case was simulated for $\mathrm{D}$ to validate the ability of the Wave microgrid controller to reconnect the microgrid to the utility grid.

\section{F. Reconnection (Test Case D1)}

The microgrid was set up to operate in islanded mode, as described in $\mathrm{C}$, except that the load and solar insolation were set to fixed values at the peak load time, similar to B1. The microgrid controller did not provide resynchronization functionality; rather, it relied on the PCC breaker to perform this function, and we therefore implemented controls in the simulated PCC breaker to perform resynchronization. The microgrid controller issued a resynchronization signal to the PCC circuit breaker to start the resynchronization process; and once the voltage, frequency, and phase angle errors between 

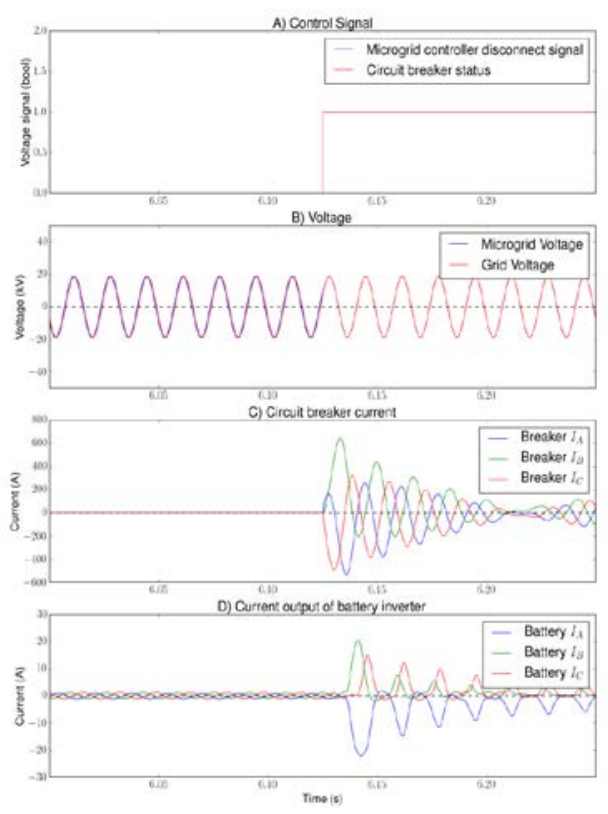

Fig. 7. Test case D1 reconnection result

the utility grid and microgrid were low enough, the PCC circuit breaker control closed the breaker.

Simulation results for D1 are shown in Fig. 7 for a few cycles before and after reconnection. Trace A shows the microgrid controller disconnect signal and the PCC circuit breaker status. The microgrid controller disconnect signal is zero, indicating that the Wave microgrid controller had previously issued a signal to reconnect. Trace B shows both the utility grid and microgrid voltages for Phase A, and Trace C shows the current through the PCC circuit breaker. Trace D shows the measured output current of the hardware battery storage inverter, scaled from the hardware rating of 540 $\mathrm{kW} / 480 \mathrm{~V} \mathrm{AC}$ to the simulated rating of $50 \mathrm{~kW} / 4.16 \mathrm{kV}$. The current transients after reconnection are larger than expected and have not been analyzed fully, but we observed that it takes some time for the controller's dispatch assignments to finalize.

\section{LESSONS LEARNED FROM HIL SIMULATION}

The proposed test methodology enabled the evaluation of the functional requirements in FOA 997. However, some of these requirements are not typically implemented by the microgrid controller itself. For example, the Wave microgrid controller relies on the synchronization relay of the PCC circuit breaker to ensure that the grid reconnection occurs when voltage, frequency, and phase angle differences between the microgrid and utility grid are small enough. We therefore recommend that microgrid controller evaluation should be performed with standard capabilities modeled in the constituent components of the microgrid. The draft version of IEEE P2030.8 is consistent with this approach.

PHIL simulation under abnormal conditions was challenging because the controllable $\mathrm{AC}$ and $\mathrm{DC}$ power supplies could trip off when they were required to operate outside the bounds of their protective settings. The appropriate settings for simulating abnormal conditions while protecting laboratory equipment are topics for further investigation.
Performance is influenced by the coefficients used in the Wave's platform's proportional integral (PI) controllers and by the design of the generator controllers we implemented. This was especially noticeable during generator mode transitionse.g., voltage/frequency master mode to baseload mode. Consequently, the generator controller design needs to be refined to more accurately represent real-world generators. We also recommend a lower ratio of real-time to simulation time steps for accelerated simulations because not all transients settled within the 2-minute simulation time step that we selected to represent 1 hour in real time.

\section{CONCLUSION}

This paper presented the development of a test bed for evaluating the functional capabilities of a microgrid controller. The test bed incorporates both CHIL and PHIL elements in the experimental setup. A campus microgrid was modeled in a digital real-time simulator, and communications were enabled among the microgrid controller hardware and the simulated assets, circuit breakers, and loads as well as with the battery inverter hardware via a PHIL interface.

It also presented a methodology for evaluating microgrid controller functionalities and described how they were tested in different scenarios. HIL simulation results provide insight into the test bed's capabilities and the types of evaluations that could be performed by using it. Such a test bed is valuable to help microgrid controller providers, microgrid developers, and owners evaluate performance prior to field deployment.

\section{ACKNOWLEDGMENT}

The authors gratefully acknowledge the contributions of Brian Miller and John Fossum to the test bed design and implementation, Dustin Schramm and Mohit Chhabra from Spirae to the CHIL interface with the Wave microgrid controller, Ben Ollis from ORNL to the test plan, and Rachna Handa from EPRI and Carol Painter from NETL to project management and oversight.

\section{REFERENCES}

[1] R. H. Lasseter, "MicroGrids," 2002 IEEE Power Engineering Society Winter Meeting. Conference Proceedings (Cat. No.02CH37309), 2002, pp. 305-308 vol.1.

[2] R. H. Lasseter and P. Paigi, "Microgrid: a conceptual solution," 2004 IEEE 35th Annual Power Electronics Specialists Conference (IEEE Cat. No.04CH37551), 2004, pp. 4285-4290 Vol.6.

[3] D. Ton and J. Reilly, "Microgrid Controller Initiatives: An Overview of R\&D by the U.S. Department of Energy," IEEE Power and Energy Magazine, vol. 15, no. 4, pp. 24-31, July-Aug. 2017.

[4] A. Maitra, A. Pratt et al., "Microgrid Controllers: Expanding Their Role and Evaluating Their Performance," in IEEE Power and Energy Magazine, vol. 15, no. 4, pp. 41-49, July-Aug. 2017.

[5] N. Ainsworth, A. Hariri, K. Prabakar, A. Pratt and M. Baggu, "Modeling and compensation design for a power hardware-in-the-loop simulation of an AC distribution system," 2016 North American Power Symposium (NAPS), Denver, CO, 2016, pp. 1-6.

[6] G. Joos, J. Reilly, W. Bower and R. Neal, "The Need for Standardization: The Benefits to the Core Functions of the Microgrid Control System," IEEE Power and Energy Magazine, vol. 15, no. 4, pp. 32-40, July-Aug. 2017. 